TRANSACTIONS OF THE

AMERICAN MATHEMATICAL SOCIETY

Volume 285, Number 1, September 1984

\title{
THE STRONG CONCLUSION OF THE F. AND M. RIESZ THEOREM ON GROUPS
}

BY

I. GLICKSBERG ${ }^{1}$

\begin{abstract}
Let $S$ be a closed proper generating subsemigroup of the dual $\Gamma$ of a locally compact abelian group $G$. Then there are Haar singular measures on $G$ orthogonal to $S$ unless $G=\mathbf{R} \times \Delta$ or $\mathbf{T} \times \Delta$ with $\Delta$ discrete, and then all $\mu$ orthogonal to $S$ are Haar absolutely continuous.
\end{abstract}

The classical F. and M. Riesz theorem $[9,10,1]$ has been extended in a variety of forms $[1-4,6]$ but almost always without the full strength of the original: an orthogonal measure has its singular component also orthogonal, but not necessarily zero. Except in the group setting there is, of course, not even a prime candidate for the singular component, but there Haar singularity is the natural choice.

Recently E. Hewitt and S. Koshi [7, 5.6, 5.3 and 5.4] have given a sharp delineation of when the strong assertion of the original prevails for measures orthogonal to half the dual group under a total (algebraic) ordering (completing an earlier result of S. Koshi and H. Yamaguchi [8]): if $G$ is a locally compact abelian group with a torsion free dual $\Gamma$, and the nondense algebraic subsemigroup $S$ totally orders $\Gamma$ (so $\Gamma=S \cup(-S)$ and $S \cap(-S)=\{0\}$ ), then there are always Haar singular measures $\mu$ on $G$ with $\hat{\mu}(S)=0$ except when $G=\mathbf{R} \times \Delta$ or $\mathbf{T} \times \Delta$ with $\Delta$ discrete; moreover, in the exceptional cases all such $\mu$ must be absolutely continuous. Both results are obtained ${ }^{2}$ as consequences of the authors' determination of all possible candidates for $S$.

Our purpose here is to show the Hewitt-Koshi-Yamaguchi results hold in the most general l.c.a. setting, with $S$ simply a subsemigoup of $\Gamma$ generating $\Gamma$ as a group (or, equivalently, separating the elements of $G$ ).

From an essentially equivalent viewpoint we are considering measures orthogonal to translation invariant subalgebras of $C_{0}(G)$ (or $C(G)$ ), and it is intriguing that our arguments for the exceptional cases seem to require both the "abstract" uniform algebra F. and M. Riesz theorem and (of course) the classical result (as extended by S. Saeki). Indeed, both our positive and negative results use the considerations of invariant subalgebras given in [5] and the existence of analytic discs in their spectra.

Received by the editors January 24, 1983 and, in revised form, June 15, 1983.

1980 Mathematics Subject Classification. Primary 46J10, 43A25.

${ }^{1}$ Work supported in part by NSF.

${ }^{2}$ The author is indebted to Professors Hewitt and Koshi for making available a preprint of [7]. Note that for convenience we take $\hat{\mu}(\gamma)=\mu(\gamma)$ for a measure (i.e. omit the usual conjugation). 
THEOREM 1. Let $G$ be a locally compact abelian group with dual $\Gamma$, and $S \subset \Gamma a$ closed proper subsemigroup which separates the points of $G$ (or equivalently, generates $\Gamma$ ). Then (1) there are Haar singular (finite) measures $\mu$ on $G$ orthogonal to $S$ unless $G=\mathbf{R} \times \Delta$ or $\mathbf{T} \times \Delta$ with $\Delta$ discrete, when (2) all such $\mu$ are Haar absolutely continuous.

The same is true if $S$ is replaced by a closed, proper, separating and translation invariant subalgebra $A$ of $C_{0}(G)$.

The proofs of assertions (1) and (2) can be read independently, in $\S 1$ and $\S 2$.

Most of what we need from [5] can be summarized as follows, where $\lambda_{z}$ represents the Poisson measure on $\mathbf{R}$ or $\mathbf{T}$ as appropriate, and $\rho^{*}$, for any map $\rho$, denotes the induced map of measures.

THEOREM 2. Suppose $S$ is a proper closed generating subsemigroup of $\Gamma$. Let $B$ be the closed span of $S$ in $C(G)$. Then:

(a) There is a continuous homomorphism $\rho$ of an open subgroup $\Gamma_{0}$ of $\Gamma$ into $\mathbf{R}$ which is nonnegative and nontrivial on $\Gamma_{0} \cap S$.

(b) With $\hat{\rho}: \mathbf{R} \rightarrow G / H$ ( $H=\Gamma_{0}^{\perp}$ compact) the dual map, and $m$ the Haar measure on $H, m * \hat{\rho} * \lambda_{z}$ provides a multiplicative linear functional $\phi_{z}$ on $B$ for each complex $z$ with $\operatorname{Im} z>0($ or $|z|<1$ if $\hat{\rho}$ is not $1-1)$.

(c) $z \rightarrow \phi_{z}$ is an analytic disc in the spectrum of $B$ (i.e. a 1-1 continuous map with $z \rightarrow \phi_{z}(b)$ analytic for each $b \in B$ ).

Finally, $\Gamma_{0}$ is proper (or $H$ nontrivial) only if $\rho$ arises from a homomorphism $\sigma$ of $\Gamma$ onto a discrete ordered abelian group $\Pi$ (with $\sigma \geqslant 0$ and nontrivial on $S$ ), followed by a nonnegative homomorphism of $\Pi_{\gamma_{0}}$ into $\mathbf{R}$ sending $\gamma_{0}$ to 1 , where $\gamma_{0}$ an arbitrary nonzero element of $\sigma S$, and $\Pi_{\gamma_{0}}$ is the subgroup of $\Pi$ of all elements bounded by some power of $\gamma_{0}$.

(Here (a) follows from [5, Theorem 2 and proof of Theorem 1], (b) and (c) from [5, Corollary 1], once we note that the existence of a nonzero measure orthogonal to $S$, hence to $B$, guarantees the strict closure of $B$ is proper, and any analytic disc in that algebra's spectrum provides one in that of $B$. The final assertion follows from the proof of $[5$, Theorem 1$]$, especially $1.7,1.3,1.4$.)

1. In proving (1) we can subsume the case of an algebra $A$ in that of a subsemigroup $S$ because of two facts: (i) $S=\Gamma \cap A^{-*}$ (the $w^{*}$ closure in $L^{\infty}(G)$ ) is a necessarily closed proper separating subsemigroup (by [5, 1.1 and 1.2]); (ii) simply replacing $\lambda_{z}$ of Theorem 2(b) above by the measure $\lambda$ (on $\mathbf{R}$ or $\mathbf{T}$ ) corresponding to a nonzero element of the usual Hardy space $H_{0}^{1}$ (of the half plane or disc) yields a nonzero measure $m * \hat{\rho} * \lambda$ orthogonal to $A$ (and $S$ ) as one easily sees, exactly as in $[5,1.7,2]$. Indeed, starting with our subsemigroup of Theorem 1 , since $\mu=m * \hat{\rho} * \lambda$ is carried by the $\sigma$-compact subgroup $H+\hat{\rho} \mathbf{R}$ of $G$, it provides our Haar singular orthogonal measure unless that subgroup is open in $G$, i.e., unless $\hat{\rho} \mathbf{R}$ is open in $G / H$. In that case $\hat{\rho}$ is either topological or the quotient map of $\mathbf{R}$ onto an open circle $\mathrm{T}$ in $G / H[12$, p. 96]. Hence we obtain (1) unless $G / H$ contains an open line or circle as a subgroup. So suppose this occurs. 
Since by structure $[\mathbf{1 0}, 12] G=\mathbf{R}^{n} \times F$, where $F$ contains an open compact subgroup $K$, and $\mathbf{R}^{n}$ has no nontrivial compact subgroup, we have $H \subset F$, so $G / H=\mathbf{R}^{n} \times(F / H)$. Thus we must have $n=1$, or $n=0$ in which case $\mathbf{T}$ is an open subgroup of $G / H=F / H$, and indeed of $K / H$. Now if $H$ is trivial we, of course, have our exceptional cases already, so we can assume $H$ is nontrivial. By Theorem 2, $H$ is nontrivial only in case $H^{\perp}$ arises as the pullback to $\Gamma$ of the subgroup $\Pi_{\gamma_{0}}$ of a totally ordered quotient $\Pi$ of $\Gamma$ consisting of those elements bounded by (some power of) a positive element $\gamma_{0}$. But $\gamma_{0}$ can be taken arbitrarily large in $\Pi$, and if powers of one $\gamma_{0}$ bound all elements of $\Pi$ we can again take $H$ trivial, so $G=\mathbf{R} \times \Delta$ or $\mathbf{T} \times \Delta$, with $\Delta$ discrete. Thus we can assume we have an increasing net $\left\{\gamma_{\alpha}\right\}$ and subgroups $\Pi_{\gamma_{\alpha}}$ whose union is $\Pi$ while $\Pi_{\gamma_{\beta}} / \Pi_{\gamma_{\alpha}}$ is infinite for $\alpha<\beta$. Correspondingly, $H_{\alpha}$ decreases to the identity (so we can take each $H_{\alpha} \subset K$ ) while $H_{\alpha} / H_{\beta}$ is infinite, and now $G / H_{\alpha}=\mathbf{R} \times F / H_{\alpha}$ with $F / H_{\alpha}$ discrete for every $\alpha$, or $G / H_{\alpha}=F / H_{\alpha}$ contains an open circle $\mathbf{T}_{\alpha}$ for every $\alpha$. In the first case $K / H_{\alpha}$ is compact and discrete, so finite, despite the fact that $\left(K / H_{\beta}\right) /\left(K / H_{\alpha}\right) \cong$ $H_{\alpha} / H_{\beta}$ is infinite for $\alpha<\beta$. In the second case $\mathrm{T}_{\alpha}$ is an open subgroup of $K / H_{\alpha}$ for every $\alpha$, so of finite index, and if $H_{\alpha} \subsetneq H_{\beta} \subsetneq H_{\delta}$ and $\sigma: K / H_{\alpha} \rightarrow K / H_{\beta}$ is the natural map, then $\mathbf{T}_{\alpha} \cap$ kern $\sigma$ is necessarily infinite, and so contains $\mathbf{T}_{\alpha}$. But that implies $K / H_{\beta}$ is finite, whence $H_{\delta} / H_{\beta}$ is, a contradiction. We now have obtained our Haar singular orthogonal measure except when $G=\mathbf{R} \times \Delta$ or $\mathbf{T} \times \Delta$, as desired.

2. Now if $G=\mathbf{R} \times \Delta$ or $\mathbf{T} \times \Delta$ and $\mu$ on $G$ is our orthogonal measure we may as well take $\Delta$ countable since we can replace $G$ by the subgroup that the support of $\mu$ generates and $A$ (or $S$ ) by the closure of its restriction to that subgroup. Moreover, $\mu$ orthogonal to $A$ implies $\mu$ is orthogonal to $S_{0}=\Gamma \cap A^{-*}$ since, for $f$ from an approximate identity in $L^{1}(G)$, we have $f * \mu$ orthogonal to $\Gamma \cap A^{-*}$, and $\hat{f}(\gamma) \neq 0$ for some $f$ depending on $\gamma \in S_{0}$. Thus we may as well consider just the case where $\hat{\mu}(S)=\mu(S)=0$ for $S$ a closed proper separating subsemigroup of $\Gamma$.

As in Theorem 2, let $B$ be the uniformly closed span of $S$ in $C(G)$. By that result we have an analytic disc in the spectrum of $B$ all of whose elements are represented by measures on a line (or circle), necessarily then coinciding with our subgroup $\mathbf{R}$ (resp. $\mathbf{T}$ ) in $G=\mathbf{R} \times \Delta$ (resp. $\mathbf{T} \times \Delta)$, since $\Delta$ is discrete; moreover, each point in the disc is represented by a measure equivalent to Lebesgue measure. By translation invariance, each coset of $\mathbf{R}$ (resp. $\mathbf{T}$ ), in fact, similarly supports an analytic disc. Indeed, since any translation (whose action on $G$ automatically extends to a homeomorphism of $M_{B}$, the spectrum of $B$ ) which maps a point in one Gleason part $P_{1}$ for $B$ [4] into a point of another, $P_{2}$, necessarily sends $P_{1}$ onto $P_{2}$; each part $P$ meeting (hence containing) one of our analytic discs necessarily contains exactly those supported by lines (or circles) lying in a coset of one fixed subgroup $G_{0}=\mathbf{R} \times \Delta_{0}\left(\right.$ resp. $\left.\mathbf{T} \times \Delta_{0}\right)$ of $G$. Let $\left.\mu\right|_{x+G_{0}}$ be the restriction of our measure $\mu$ to the coset $x+G_{0}$.

Since $G$ maps in a 1-1 continuous fashion into the Silov boundary $\partial_{B}$ of the spectrum of $B$, our measures on $G$ can be taken as measures on $\partial_{B}$. For our $\mu$ on $G$ orthogonal to $B$, if $\left\{f_{j}\right\}$ is an approximate identity in $L^{1}(\mathbf{R} \times\{0\})$ (resp. $\left.L^{1}(\mathbf{T} \times\{0\})\right) \subset L^{1}(G)$ then $f_{j} * \mu(\gamma) \rightarrow \mu(\gamma)$ for any $\gamma \in \Gamma$. But each measure $f_{j} * \mu$ 
is absolutely continuous, and thus, its absolutely continuous component relative to the Gleason part [4, p. 142], containing our discs supported by $x+G_{0}$, is precisely $\left.\left(f_{j} * \mu\right)\right|_{x_{0}+G_{0}}=f_{j} *\left(\left.\mu\right|_{x_{0}+G_{0}}\right)$, as we see from the following argument.

By a result of Bishop [4, p. 143] each point $\phi$ in one disc has a representing measure dominating our Possion measure representing an element of another disc in our part; since $\Delta$ is countable, $\phi$ thus has a representing measure dominating Haar measure on $x+G_{0}$. Hence $\left.\left(f_{j} * \mu\right)\right|_{x_{0}+G_{0}}$ is absolutely continuous relative to our part. But, because these measures for the various distinct cosets $x_{0}+G_{0}$ are mutually singular, and sum to $f_{j} * \mu$, they must yield our part components, as asserted.

Now by the abstract F. and M. Riesz theorem [4, p. 44] $\left.\left(f_{j} * \mu\right)\right|_{x_{0}+G_{0}}$ is still orthogonal to $B$, hence $S$. Consequently $\left.\mu\right|_{x_{0}+G}(\gamma)=\lim f_{j} *\left(\left.\mu\right|_{x_{0}+G_{0}}\right)(\gamma)=0$ for $\gamma \in S$.

Clearly it will suffice to show each $\left.\mu\right|_{x+G_{0}}$ is absolutely continuous, and without loss of generality we may assume $\left.\mu\right|_{G_{0}} \neq 0$ and show it is absolutely continuous. Thus we may as well replace $G$ by $G_{0}$, in effect replacing $S$ by its image in the quotient $\Gamma / G_{0}^{\perp}=\hat{G_{0}}$ and $B$ by $\left(B \mid G_{0}\right)^{-}$, both of which $\left.\mu\right|_{G_{0}}$ annihilates, while our cosets of $\mathbf{R} \times\{0\}$ (resp. $\mathbf{T} \times\{0\}$ ) still bound analytic discs all lying in one part $P$ for $\left(B \mid G_{0}\right)^{-}$. Hence we may as well assume $G=G_{0}$.

We shall first show $G=G_{0}$ reduces to our open line or circle, and then that $\mu=\left.\mu\right|_{G_{0}}$ is absolutely continuous. Since $G=G_{0}=\mathbf{R} \times \Delta_{0}\left(\right.$ or $\left.\mathbf{T} \times \Delta_{0}\right)$ with $\Delta_{0}$ countable, our dual $\Gamma=\mathbf{R} \times K$ (or $\mathbf{Z} \times K$ ), with $K$ compact and metric; let $\rho$ denote a translation invariant metric on $K$. Supposing $\Delta_{0}$ is nontrivial, $K$ is not, and fixing $k_{0} \in K, k_{0} \neq 0$, we have $K_{0}=\left(\mathbf{Z}_{+} k_{0}\right)^{-}$, as a closed subsemigroup of $K$, actually a closed nontrivial subgroup.

Now for any $\delta>0$ we have an $n=n_{\delta}$ for which $\left\{k_{0}, 2 k_{0}, \ldots, n k_{0}\right\}$ is $\delta$-dense in $K_{0}$. Because the subgroup $S-S$ must be dense in $\Gamma=\mathbf{R} \times K($ resp. $\mathbf{T} \times K)$, hence has $\{0\} \times K$ in its closure, we can find $(r, k),\left(r^{\prime}, k^{\prime}\right)$ in $S$ with

$$
\left|r-r^{\prime}\right|<\delta / n, \quad \rho\left(k, k_{0}\right)<\delta / 3 n, \quad \rho\left(k^{\prime}, 0\right)<\delta / 3 n .
$$

(To see this, first choose $\left(r_{1}-r_{2}, k_{1}-k_{2}\right) \in S-S$ close to $\left(0, k_{0}\right)$, and $\left(r_{3}-r_{4}\right.$, $\left.k_{3}-k_{4}\right) \in S-S$ close to $(0,0)$. Then $\left(r_{1}+r_{4}, k_{1}+k_{4}\right)$ is close to $\left(r_{2}+r_{3}, k_{2}+\right.$ $\left.k_{3}\right)+\left(0, k_{0}\right)$, and because $\left(\pi_{2} S\right)^{-}$is necessarily $K$ (as a closed subsemigroup, hence a subgroup, which separates the dual $\left.\Delta_{0}\right)$ we have $\left(r_{5}, k_{5}\right) \in S$ with $k_{5}$ close to $-\left(k_{2}+k_{3}\right)$. Thus $\left(r_{2}+r_{3}+r_{5}, k_{2}+k_{3}+k_{5}\right)=\left(r^{\prime}, k^{\prime}\right)$ is close to $\left(r^{\prime}, 0\right)$ whence $\left(r_{1}+r_{4}+r_{5}, k_{1}+k_{4}+k_{5}\right)=(r, k)$ is a close to $\left(r^{\prime}, 0\right)+\left(0, k_{0}\right)=\left(r^{\prime}, k_{0}\right)$.)

Now for every choice of $\eta=\left(\eta_{1}, \eta_{2}, \ldots, \eta_{n}\right)$ with $\eta_{j}=0$ or 1

$$
\gamma_{\eta}=\left\{\sum_{j=1}^{n}\left(\eta_{j} r+\left(1-\eta_{j}\right) r^{\prime}\right), \sum_{j=1}^{n}\left(\eta_{j} k+\left(1-\eta_{j}\right) k^{\prime}\right)\right\} \in S
$$

while $\left|\pi_{1} \gamma_{\eta}-n r\right|<\delta$, so that

$$
\{n r\} \times K \subset \bigcup_{\eta} B\left(\gamma_{\eta}, \delta\right)
$$

where $B\left(\gamma_{\eta}, \delta\right)=\left\{\gamma=(r, k):\left|r-\pi_{1} \gamma_{\eta}\right|<\delta, \rho\left(k, \pi_{2} \gamma_{\eta}\right)<\delta\right\}$. 
Since $K_{0}$ is a nontrivial subgroup of $K, K_{0}^{\perp}$ in $\Delta_{0}=K^{\wedge}$ is proper. Let $d \in \Delta_{0} \backslash K_{0}^{\perp}$, and let $\lambda_{1}$ be a measure on $\mathbf{R} \times\{0\}$ (resp. $\mathbf{T} \times\{0\}$ ) representing a point in our part $P, \lambda_{2}$ a measure in $\mathbf{R} \times\{d\}$ (resp. $\mathbf{T} \times\{d\}$ ) representing a point in $P$. Since $\lambda_{1}$ and $\lambda_{2}$ have uniformly continuous Fourier-Stieltjes transforms we can, for any $\varepsilon>0$, choose $\delta>0$ so that $\left|r_{1}-r_{2}\right|<\delta, \rho\left(k_{1}, k_{2}\right)>\delta$ imply $\left|\hat{\lambda}_{i}\left(r_{1}, k_{1}\right)-\hat{\lambda}_{i}\left(r_{2}, k_{2}\right)\right|<\varepsilon$. For the corresponding $n=n_{\delta}$ and $\gamma_{\eta}$ in (2), if $\left\{E_{1}, E_{2}, \ldots, E_{s}\right\}$ is a partition of $K$ with $\{n r\} \times E_{j} \subset B\left(\gamma_{\eta(j)}, \delta\right)$ for some $\eta(j)$, then for $m_{0}$ the Haar measure of $K_{0}$ we have

$$
\left|\sum_{j=1}^{s} m_{0}\left(E_{j}\right) \hat{\lambda}_{i}\left(\gamma_{\eta(j)}\right)-\int \hat{\lambda}_{i}(n r, k) m_{0}(d k)\right|<\varepsilon, \quad i=1,2 .
$$

Since $\int \hat{\lambda}_{i} d m_{0}=\int \hat{m}_{0} d \lambda_{i}=\delta_{i 1}$, this says the element $b=\sum_{j=1}^{s} m_{0}\left(E_{j}\right) \gamma_{\eta(j)}$ of $B$, which lies in the unit ball since $\sum_{j=1}^{s} m_{0}\left(E_{j}\right)=1$, has $\left|\lambda_{1}(b)-1\right|<\varepsilon,\left|\lambda_{2}(b)\right|<\varepsilon$. Because $\varepsilon>0$ is arbitrary, that says $\lambda_{1}$ and $\lambda_{2}$ represent points in distinct Gleason parts, our contradiction, showing $\Delta_{0}$ is indeed trivial, so $G_{0}=\mathbf{R} \times\{0\}$ (or $\mathbf{T} \times\{0\}$ ). (In fact, we see that when $G$ has $\mathbf{R}$ or $\mathbf{T}$ as an open subgroup, our analytic discs are parts.)

Now we know the restriction of $\mu$ to each coset of $G_{0}=\mathbf{R} \times\{0\}$ or $\mathbf{T} \times\{0\}$ is orthogonal to $S$. Considered as a measure on $\mathbf{R}$ or $\mathbf{T},\left.\mu\right|_{G_{0}}$ has a Fourier-Stieltjes transform vanishing on the subsemigroup $\pi_{1} S$ of $\mathbf{R}$ or $\mathbf{Z}$, which necessarily separates the dual group $\mathbf{R}$ or $\mathbf{T}$. In the second case it is now trivial to see $\left.\mu\right|_{G_{0}}$ is absolutely continuous since $\pi_{1} S$ necessarily lies in $\mathbf{Z}_{+}$(or $\mathbf{Z}_{-}$) and contains a pair $p, q$ of relatively prime integers $p, q$, indeed with $p-q=1$ since the subgroup $\pi_{1} S-\pi_{1} S$ of $\mathbf{Z}$ cannot be proper. Thus $\pi_{1} S$ contains all $n \geqslant q^{2}+q$ since $n=q Q+r=q Q+$ $r(p-q)=q(Q-r)+r p$ where $0 \leqslant r<q$, and $Q-r>0$ if $Q=(n-r) / q>$ $(n-q) / q$ exceeds $q>r$, i.e. if $n-q>q^{2}$. Now the classical result says $\left.\mu\right|_{G_{0}}$ is absolutely continuous.

In case $G_{0}=\mathbf{R} \times\{0\}$ we again have $\pi_{1} S \subset \mathbf{R}_{+}$(or $\mathbf{R}_{-}$) and since $\pi_{1} S$ separates the dual line, it is necessarily dense modulo 1 (again because a closed subsemigroup of a compact group is a subgroup). But S. Saeki showed (in 1977) that if $B \subset \mathbf{R}$ is dense modulo 1 and a measure $\mu$ on $\mathbf{R}$ has $\hat{\mu}(n+b)=0$ for all $b \in B$ and $n \in \mathbf{Z}_{+}$ then $\mu$ is absolutely continuous; appropriately dilating our line and its dual we can take $1 \in \pi_{1} S$, so Saeki's result applies to yield the desired conclusion and our proof is complete.

I am indebted to Professor Saeki for the following proof of his result. (For related but deeper results, see [11].) Let $\delta_{x}$ denote the point mass at $x, \nu_{a}, \nu_{s}$ the absolutely continuous and singular components of a measure $\nu$, and $M_{s}$ the space of all singular measures. Fix $b \in B$, and let $\nu=e^{i b \cdot} \mu$, so $\hat{\nu} \mid \mathbf{Z}_{+}=0$ by hypothesis. We claim $\hat{\boldsymbol{v}}_{s} \mid \mathbf{Z}=0$ as a consequence. Indeed we trivially have

$$
\nu=\sum_{k=-\infty}^{\infty} \nu_{k} * \delta_{2 \pi k}
$$

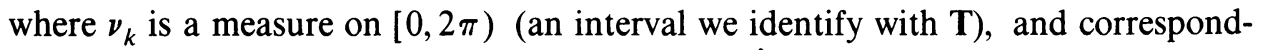
ingly $\nu_{a}=\Sigma\left(\nu_{k}\right)_{a} * \delta_{2 \pi k}, \nu_{s}=\Sigma\left(\nu_{k}\right)_{s} * \delta_{2 \pi k}$. Since $\hat{\delta}_{2 \pi k}(\mathbf{Z})=1$ we thus obtain

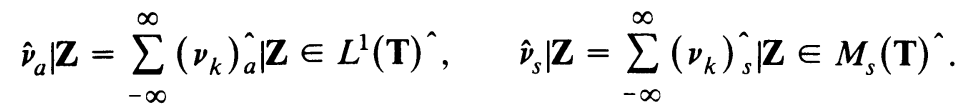


But $\hat{\nu}\left(\mathbf{Z}_{+}\right)=0$ implies $\hat{\nu} \mid \mathbf{Z} \in L^{1}(\mathbf{T}){ }^{\wedge}$ by the classical F. and M. Riesz Theorem, so

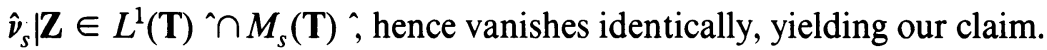

Now we have $\hat{\mu}_{s}(b+n)=\hat{\nu}_{s}(n)=0$ for any $n \in \mathbf{Z}$, and since $b \in B$ is arbitrary, $\hat{\mu}_{s}$ vanishes on $B+\mathbf{Z}$, which is dense in $\mathbf{R}$ since $B$ is dense modulo 1 . We conclude $\hat{\mu}_{s} \equiv 0$, whence $\mu_{s}=0$ as desired.

REMARK. The portion of the preceding argument reducing a part to a single disc shows something more general. Call the closure of the union of the supports in $G$ of the representing measures for points in a Gleason part $P$ the support of $P$. Then for any closed proper separating translation invariant subalgebra of $C_{0}(G), G$ l.c.a., the support of a part $P$ is connected. By translation invariance the support is a coset of a subgroup $G_{0}$, and we want to see $G_{0}$ is connected. But if that fails $G_{0}$ has an open proper subgroup $G_{1}$, so $G_{0} / G_{1}$ is discrete, and reducing our considerations to the closed span $B$ of $S_{0}=\Gamma \cap A^{-*}$ in $C(G)$, and $\left(B \mid G_{0}\right)^{-}$as before (with $P$ remaining in one part), we can use the nontrivial compact group $G_{1}^{\perp}$ in $\hat{G_{0}}$ for $K$ and argue as before to a contradiction.

Finally we might note that without separation there are discrete orthogonal measures, so that (1) holds trivially unless $G$ is discrete; on the other hand for $G$ discrete, all measures are absolutely continuous, but no $S$ or $A$ satisfying the hypotheses of Theorem 1 can exist (for $S$ is necessarily a group).

\section{REFERENCES}

1. K. deLeeuw and I. Glicksberg, Quasi-invariance and measures on compact groups, Acta Math. 109 (1963), 179-205.

2. F. Forelli, Analytic and quasi-invariant measures, Acta Math. 118 (1967), 33-59.

3. __ Measures orthogonal to polydisc algebras, J. Math. Mech. 17 (1968), 1073-1086.

4. T. W. Gamelin, Uniform algebras, Prentice-Hall, Englewood Cliffs, N. J., 1969.

5. I. Glicksberg, Spectra of invariant uniform and transform algebras, Trans. Amer. Math. Soc. 277 (1983), 381-396.

6. H. Helson and D. Lowdenslager, Prediction theory and Fourier series in several variables, Acta Math. 99 (1958), 165-202.

7. E. Hewitt and S. Koshi, Orderings in locally compact abelian groups and the Theorem of $F$. and $M$. Riesz, Math. Proc. Cambridge Philos. Soc. 93 (1983), 441-457.

8. S. Koshi and H. Yamaguchi, The F. and M. Riesz theorem and group structures, Hokkaido Math. J. 8 (1979), 294-299.

9. F. Riesz and M. Riesz, Über die Randwerte einer Analytishcen Functionen, (4 ${ }^{e}$ Congrès des Math. Scand.), 1916, p. 27-44.

10. W. Rudin, Fourier analysis on groups, Interscience, New York, 1962.

11. S. Saeki, The F. and M. Riesz Theorem and singular measures (to appear).

12. A. Weil, L'intégration dans les groupes topologiques et ses applications, Actualités Sci. Indust., no. 869, Paris, 1940.

Department of Mathematics, Univeritity of Washington, Seattle, Washington 98195 